\title{
An Orthogonal Wavelet Transform-Based K-Nearest Neighbor Algorithm to Detect Faults in Bearings
}

\author{
Weipeng Li $\mathbb{D}^{1,2}$ Yan Cao $\mathbb{D D}^{1}{ }^{1}$ Lijuan Li $\mathbb{D}^{1},{ }^{1}$ and Siyu Hou $\mathbb{D}^{1}$ \\ ${ }^{1}$ School of Mechanical Electrical Engineering, Xi'an Technological University, Xi'an 710000, China \\ ${ }^{2}$ School of Intelligent Manufacturing, Nanyang Institute of Technology, Nanyang 473000, China \\ Correspondence should be addressed to Yan Cao; caoyan@xatu.edu.cn
}

Received 9 November 2021; Revised 13 January 2022; Accepted 1 February 2022; Published 18 February 2022

Academic Editor: Xavier Chiementin

Copyright (c) 2022 Weipeng Li et al. This is an open access article distributed under the Creative Commons Attribution License, which permits unrestricted use, distribution, and reproduction in any medium, provided the original work is properly cited.

We aim to address the issues of difficult acquisition of bearing fault data, few feature data sets, and low efficiency of intelligent diagnosis. In this paper, an orthogonal wavelet transform K-nearest neighbor (OWTKNN) diagnosis method has been proposed. The (OWT) method extracts the peaks of each detail signal as training samples and uses the K-Nearest Neighbor (KNN) method for fault classification. The classification results of the multiple fault test data obtained through rolling bearing tests show that the method can reach a fault recognition rate of $100 \%$, and compared with KNN without extracted eigenvalues, it significantly improves the classification effects from various unknown fault data of the bearing inner ring and ball, shortens classification time, and improves the intelligent diagnosis efficiency. In addition, it achieves an overall recognition rate exceeding 95\%, Comparing OWT, EMD, and VMD feature extraction methods, both the OWTKNN and k-center point clustering algorithm do not exceed $80 \%$ (KCA), also bearing testimony of the effectiveness of this method.

\section{Introduction}

Fault intelligent diagnosis and identification are an important reason to develop machine learning methods $[1,2]$ and apply them in mechanical fault diagnosis technology $[3,4]$ in recent years. In supervised learning $[5,6]$, the process of using a set of samples from a known class to adjust the parameters of a classifier to achieve the required performance is also known as supervised training or teacherguided learning. The supervised learning algorithm is a function that analyzes this training data and produces an inference that can be used to map new instances. An optimal solution will allow the algorithm to correctly determine class labels for those unseen instances. K-Nearest Neighbor (KNN) [7-9], originally proposed by Cover and Hart in 1968 , is a comparatively theoretically mature classification algorithm. It is a commonly used supervised learning method with a very simple mechanism: when a test sample is given, find the $k$ training samples that are near it in the set based on distance measurements, and then make predictions based on the information of these $k$ "neighbors." Those methods that learn the samples during the training phase are called "eager learning" [10]. However, in engineering applications, since most fault signals have rough signals and positive and negative data, traditional signal processing methods are not applicable, and if the collected signals are directly used in the K-Nearest Neighbor algorithm for fault diagnosis, incorrect classifications will occur. On the other hand, the KNN algorithm is better suited for automatically classifying the class domain with large sample sizes, while those with small sample sizes are prone to misclassifications. Therefore, using an appropriate method for signal feature extraction not only reflects the feature information contained in the signal more accurately, it also greatly reduces data dimensions so that the KNN algorithm can quickly and accurately give fault diagnostic results. KNN is extremely effective in classifying vibration signals from faults in bearings, analyzing and classifying angle grinder working conditions, and analyzing acoustic signals with infrared (IR) thermal imaging and infrared image processing technology, solidifying its promise as a tool that can improve accuracy in fault diagnosis [11-13]. 
Wavelet transform is an emerging branch of applied mathematics that was developed in the late 1980s. Wavelet transform has multiresolution characteristics (multiscale characteristics) that can gradually observe coarse and fine signals. Its multiresolution properties also manifest through its "zoom" feature, which is crucial for localized analyses of nonstationary signals $[14,15]$. Feature extraction does possess a critical bottleneck because noiseless weak fault signals directly affect the accuracy, effectiveness, or abnormal values of whole-body vibration fault diagnosis in aero-engines. When predicting the service lives of key bearing components in fault diagnosis $[16,17]$, it is crucial to reflect the running state [18] of the system through extracted features and remain highly sensitive to abnormal signals, and the wavelet entropy theory analysis technology that has been proposed can effectively process vibration signals for general industrial equipment. However, since fault signals are prone to interference from noise and outliers, it is impractical to completely rely on the wavelet entropy method to diagnose whole-body vibration faults in aero-engines $[19,20]$. The orthogonal wavelet transform is a wavelet transform with an orthogonal wavelet function that can characterize the local signal attributes in both the time and frequency domains, which can decompose the original signal into the corresponding local details at each scale. Analyzing these local detail signals can more effectively acquire attributed information from the original data. Therefore, this paper proposes combining orthogonal wavelet transform (OWT) [21, 22] with the KNN algorithm [23, 24], and this Orthogonal Wavelet Transform K-Nearest Neighbor (OWTKNN) is applied to fault diagnosis. The OWT method extracts peak-to-peak signals of each detail as training samples of the KNN algorithm, and learning the unlabeled training samples reveals the intrinsic properties and laws of the data. The results show the following: (1) Compared with KNN without extracted eigenvalues, this method can effectively process complex mechanical vibration signals. (2) Obviously improve the classification effect of various fault data of the bearing inner ring and the ball, shorten the classification time, and improve the efficiency of intelligent diagnosis. (3) By changing the features of different layers of orthogonal wavelet. (4) Compared with the K center clustering algorithm (KCA), the effectiveness of this method is also proved [25-27].

The innovations and main contributions of this paper are described as follows [28-30]:

(1) This paper proposes a method combining the Orthogonal Wavelet Transformation (OWT) and K-Nearest Neighbor (KNN)

(2) The orthogonal wavelet transform refers to the selection of orthogonal wavelet function for the wavelet transform to be able to characterize the local characteristics of the signal in both the time domain and the frequency domain

(3) The Orthogonal Wavelet Transformation (OWT) method is used to extract the peak-to-peak value of each detail signal as a training sample for OWTKNN fault classification
(4) The effectiveness of the OWTKNN method of the method has been verified by comparing it with the commonly used signal feature extraction methods EMD and VMD

\section{K-Nearest Neighbor Algorithm}

2.1. Principles of the K-Nearest Neighbor Algorithm. The K-Nearest Neighbor (KNN) classification algorithm, a theoretically mature method, is also one of the simplest machine learning algorithms. The idea behind this method is that if the majority of the $\mathrm{k}$ nearest (i.e., the closest neighbors in the feature space) samples in the vicinity of a sample belong to a certain class, the sample also belongs to that class $[31,32]$. That is, given a training dataset, for a new input instance, find the $k$ nearest instances to the instance in the training dataset (i.e., the $k$ neighbors mentioned above), and if the majority of these $k$ instances belong to a certain class, classify the input instance into this class. As shown in Figure 1, if $k=3$, the nearest 3 neighbors of the green dot are 2 red triangles and 1 blue square. The minority is subordinate to the majority. Based on the statistical method, the green point will be classified as a red triangle class. If $k=5$, the nearest 5 neighbors of the green dot are 2 red triangles and 3 blue squares; the minority is still subordinate to the majority. Based on the statistical method, it is determined that this green point to be classified is subordinate to the class of blue squares. When it is not possible to determine which class the current point to be classified is subordinate to, we can look at its location characteristics based on the statistical theory, measure the weights of its surrounding neighbors, and classify it as (or assign it to) the class with the greater weights. This is the core concept of the KNN algorithm.

2.2. Characteristics of the KNN Algorithm. The advantages of the KNN algorithm are as follows:

(1) Simple, effective, low recurrence, no parameter estimation, no training.

(2) Highly accurate, unaffected by noise.

(3) Since the KNN method primarily relies on a limited number of neighboring samples, rather than on the discriminative class domain to determine its class, it is more suitable than other methods for a set of samples to be assigned with more intersection or overlap of class domains.

(4) It is especially suitable for multiclassification problems and features a superior SVM performance.

The disadvantages of the KNN algorithm are as follows:

(1) For computing classifications, the computational volume is large, as each sample to be classified has to calculate the distance with all known samples to get the $k$ nearest neighbors.

(2) Poor interpretability, as it cannot be explained as effectively as the decision tree algorithm. 


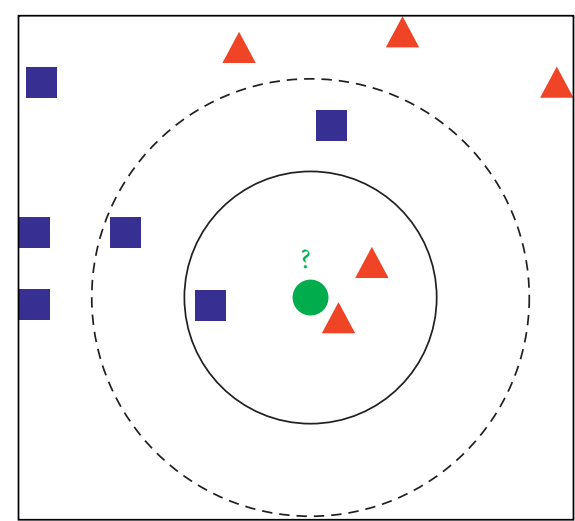

FIgURE 1: Example of the KNN method.

(3) When the samples are unbalanced, if the sample size of one class is large and the other sizes are small, it is possible that when a new sample is an input, the class will account for the majority of the $k$ neighboring samples of that sample.

(4) This algorithm is suitable for automatically classifying classes with large sample sizes. If this algorithm is applied when small sample sizes are presented, it is prone to misclassification.

The choice of $k$ values will significantly impact the algorithm results. A smaller value means that only the training instance that is closer to the input instance can contribute to the prediction results, but overfitting easily occurs. Although a larger value can reduce estimation errors while learning, the disadvantage is that the approximate errors when learning increase. Training instances far away from the input instance will still affect predictions, making those predictions unreliable. When practically applied, the value of $k$ is usually a smaller value, and the intersection and verification method is usually used to determine the optimal value of $k$ [33].

The original KNN algorithm is shown in equation (1), and after the weights are introduced, the distance formula is shown in formula (2).

$$
\begin{aligned}
& d_{i j}=\left\{\sum_{h=1}^{n}\left(a_{i h}-a_{j h}\right)^{2}\right\}^{1 / 2}, \\
& d_{i j}=\left\{\sum_{h=1}^{n} \omega_{h}\left(a_{i h}-a_{j h}\right)^{2}\right\}^{1 / 2} .
\end{aligned}
$$

In the formula, $d_{i j}$ represents the distance between sample $i$ and sample $j$; n represents the total number of attributes; $a_{i j}$ represents the $h$-th attribute in sample $i$; the $\omega_{h}$ represents the weight of the $h$-th attribute. By introducing the weights, one can act as an attribute equilibrium, similar to normalization processing. To overcome the unreasonable situation caused by the weight, each class of weight formula is shown in equation (3). After introducing the similarity parameter, the weight calculation formula is shown in equation (4).

$$
\begin{aligned}
& p\left(x, C_{j}\right)=\sum_{i=1}^{k} p_{a}\left(a_{i}, C_{j}\right), \\
& p\left(x, C_{j}\right)=\sum_{i=1}^{k} \operatorname{Sim}\left(a_{i}, x\right) p_{a}\left(a_{i}, C_{j}\right) .
\end{aligned}
$$

Suppose that the $k$ nearest neighbor samples of sample $x$ to be classified are collectively divided into the $j$ class, where $p\left(x, C_{j}\right)$ indicates the sample $x$ to be classified. A weight is belonging to class $j$. $\operatorname{Sim}\left(a_{i}, x\right)$ represents the resemblance between the nearest neighbor sample $a_{i}$ and $x$, which can be expressed as the reciprocal of the European distance between $a_{i}$ and $x$.

\section{Orthogonal Wavelet Transformations}

Wavelet bases are not necessarily orthogonal ones in wavelet transformations, but finding orthogonal wavelet bases is desired in practical applications. The important method for constructing the orthogonal wavelet basis is the multiscale analysis. When the orthogonal wavelet basis is chosen for wavelet transformation, it can decompose the original signals in both time and frequency one by one into the corresponding local detail signals at each scale. By analyzing the local detail signals, one can capture characteristic information from the original data more effectively. The number of orthogonal wavelet decomposition layers, the scale corresponding to oneto-one, and the number of decomposition layers have several scales. Scaling is like using a ruler, and each decomposition layer measures the original signal with a different ruler. Therefore, there are a few decomposition layers with a few rulers. However, the analysis frequency determines the number of decomposition layers. Thus, a finer ruler is required to measure the signal, only related to the original signal data quantity, independent of the sampling frequency, frequency, and sampling period [34].

The expansion of function $z(t)$ in an arbitrary space $L^{2}(R)$ under a wavelet basis is called the wavelet transform of function $z(t)$ (abbreviated as $W T$ ) [35], and its expression is as follows:

$$
\begin{aligned}
W T_{z}(a, b) & =\left\langle z(t), \phi_{a, b}(t)\right\rangle \\
& =\frac{1}{\sqrt{a}} \int_{R} z(t) \phi\left(\frac{t-b}{a}\right) \mathrm{d} t .
\end{aligned}
$$

The wavelet basis in wavelet transform is not necessarily an orthogonal basis, but in practical applications, it prefers to find an orthogonal wavelet basis. An important method for constructing orthogonal wavelet bases is called multiscale analysis.

The multiscale analysis is strictly defined by function space terms, assuming that the subspace sequence $V_{m}(m \in Z)$ in the space $L^{2}(R)$ satisfies the following conditions:

(1) (nestedness) : $\ldots V_{2} \subset V_{1} \subset \ldots \subset V_{-2} \subset \ldots$

(2) (approximation property) : $\bigcap_{m \in z} V_{m}=\{0\}, \underset{m \in z}{\cup} V_{m}=$ $L^{2}(R)$. 
(3) (scalability) : $f(t) \in V_{n} \Leftrightarrow f(2 t) \in V_{m-1}$.

(4) $V_{m}=\operatorname{span}\left\{\varphi_{m, n}(t), n \in Z\right\}$, i.e., any level of subspace can be formed by the same function of the corresponding scale by translation.

(5) $V_{m-1}=V_{m} \oplus W_{m}$, that is, any level of subspace can be summarized by the next level of subspace and its orthogonal complement space, and the sequences $W_{m}$ do not overlap with each other and are orthogonal systems.

Thus, we call the subspace sequences $V_{m}$ and $W_{m}$ multiscale analyses of the function space. Among them, $\varphi_{m, n}$ is called the scale function, and $m$ and $n$ are the scale and translation parameters, respectively.

From the aforementioned definitions, we have the following:

$$
V_{0}=V_{m} \oplus \sum_{i=1}^{m} W_{i} .
$$

The application of multiscale analyses in signal processing can be expressed by equation (6). The arbitrary function $f(t) \in V_{0}$ can be decomposed on the next level of scale space $V_{1}$ and wavelet space $W_{1}$, as follows:

$$
f(t)=p_{1} f(t)+q_{1} f(t)
$$

where $p_{1} f(t)=\sum_{k} C_{1, k} \varphi_{1, k}$ and $q_{1} f(t)=\sum_{k} D_{1, k} \theta_{1, k}$.

$p_{1} f(t)$ is the approximation part, and $q_{1} f(t)$ is the detail part. Then, the approximation part is further decomposed repeatedly to obtain the approximation and detail parts on any scale. The iterative formula is as follows:

$$
\begin{aligned}
p_{m-1} f(t) & =p_{m} f(t)+q_{m} f(t) \\
& =\sum_{k} C_{m, k} \varphi_{m, k}+\sum_{k} D_{m, k} \theta_{m, k},
\end{aligned}
$$

where $C_{m}=H C_{m-1}$ and $D_{m}=G C_{m-1} . H$ is the low-pass filter. For each decomposition, the sampling of $p_{m} f(t)$ is twice as sparse as the original, and the resolution is getting coarser while the waveform is getting smoother. $G$ is the mirror high-pass filter of $H$, and the bandwidth is also reduced by a factor of two each time. After $m$ decompositions, we have the following:

$$
f(t)=p_{m} f(t)+\sum_{j=1}^{m} q_{j} f(t) .
$$

The formula is the low-frequency global information of the function, and the second term is the corresponding local detail information of $f(t)$ on each scale from $V_{0}$ to $V_{m-1}$, obtained by successive decompositions [36], and the aforementioned decompositions are shown in Figure 2.

\section{Orthogonal Wavelet Transform KNN Multiclassification Diagnosis Method}

The aforementioned orthogonal wavelet transform is used to decompose vibration signals, extract the features for the corresponding local signal $f(t)$ on each scale, extract the

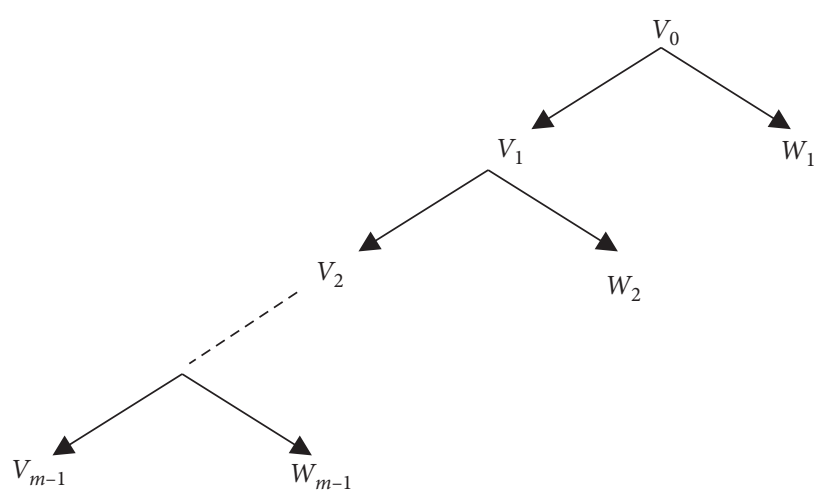

FIgURE 2: Orthogonal wavelet analysis.

local signals as a feature vector, and then use the KNN algorithm to classify any faults. This can achieve intelligent fault diagnosis [37]. The specific method is below.

Step 1. The original signal is subjected to an $m$-layer orthogonal wavelet transform, and the corresponding local detail signals of $f(t)$ on each scale from $V_{0}$ to $V_{m-1}$ are extracted, which are analyzed as seen in Figure 2, where $V_{0}$ is the original signal.

Step 2. The peak-to-peak values of the signals on each scale are founded, and the feature vector is constructed. Let the corresponding peak-to-peak value of $W_{i} \quad(i=1,2, \ldots, m-1)$ be $\operatorname{Xpp}(i)=\max \left(W_{i}\right)-\min \left(W_{i}\right), \quad(i=1,2,3 \ldots, m-1)$, and the feature vector $T$ is constructed as follows:

$$
T=[\operatorname{Xpp}(1), \operatorname{Xpp}(2), \operatorname{Xpp}(3), \ldots, \operatorname{Xpp}(m-1)] .
$$

Step 3. Use the feature vectors constructed in Step 2 as learning samples to construct the classification model and train the KNN classifier.

Step 4. The trained KNN multiclassifier is used to classify fault signals.

The steps for the OWTKNN algorithm are shown in the flowchart in Figure 3.

\section{Experimental Analysis}

5.1. Experimental Conditions. The bearing fault data from the electrical engineering laboratory of Case Western Reserve University, USA, on the rolling bearing experimental bench in Figure 4 were analyzed [38] to validate the effectiveness of the OWTKNN multiclassification diagnostic method. The bearing model was 6205-2RS deep groove ball bearing, which was mounted on the drive end of the right side of the motor to support the motor shaft. The rolling bearing multichannel fault signal was synchronously collected by three acceleration sensors, and the sensor installation positions are shown in Figure $4[39,40]$. The sampling frequency was $12 \mathrm{kHz}$, and the sampling length was 512 points in the normal state of the bearing. Two sets of normal data were collected at a bearing rotation speed of $1797 \mathrm{r} / \mathrm{min}$ and under 0 HP load and at a bearing rotation speed of 1772 


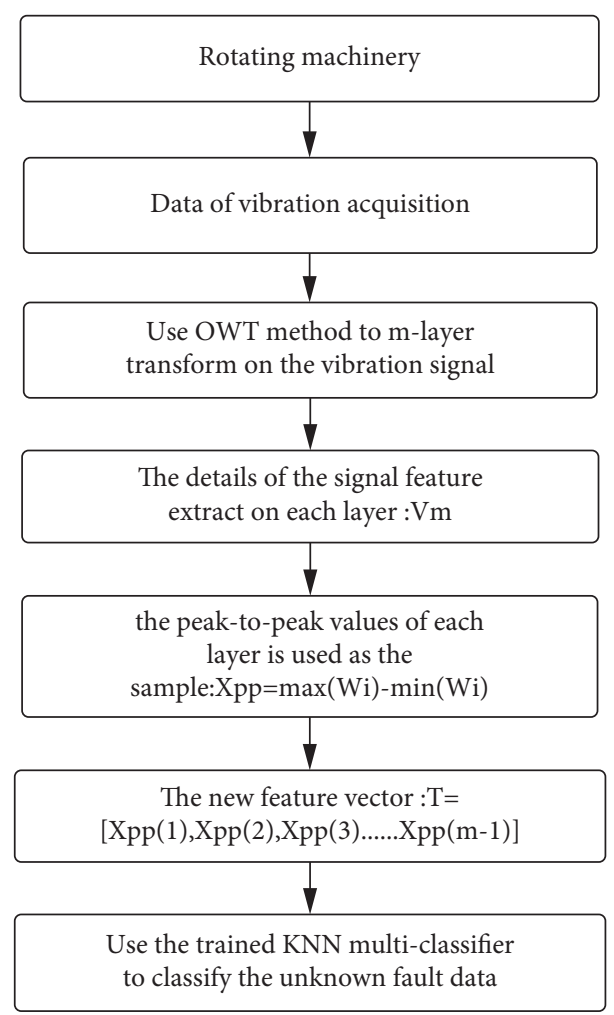

FIgURE 3: Flow chart of OWTKNN.

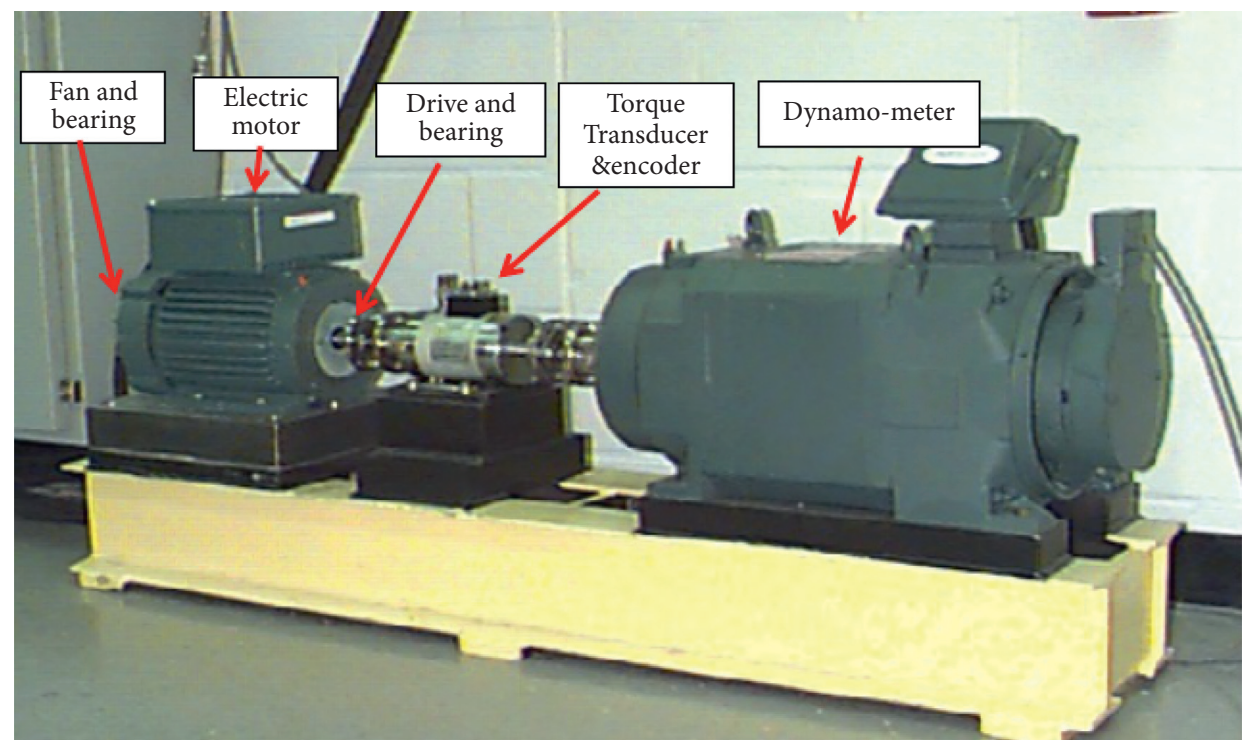

FIGURE 4: Rolling bearing test bench.

r/min and under 1 HP load, respectively. Using electrical discharge machining (EDM) for motor bearing fault implantations, a fault with a diameter of 0.007 in. It was introduced between the inner track and the ball. 4 sets of data were collected for $0 \mathrm{HP}$ and $1 \mathrm{HP}$ load under the same sampling conditions, with the inner ring and ball faults each having 2 sets of data. For the fault with a diameter of 0.014 in., another 4 sets of data were also collected for $0 \mathrm{HP}$ and 1 HP loads under the same conditions, with the inner rings and ball faults each having 2 sets of data. As a result, 8 sets of data were collected in total. The eight sets of data collected were used as input vectors for the OWTKNN multiclassifier to test fault signal classification. Figures 5 and 6 show the waveforms after 5 layers of orthogonal wavelet decomposition when the bearing under $0 \mathrm{HP}$ load was normal and in the inner ring fault (0.007 in.), respectively. From the figures, it can be seen that as the decomposition layers increased, the local signal's regularity increased, which was especially noticeable in the second and third layers. With the increase in decomposition layers, nonstationary signals were 


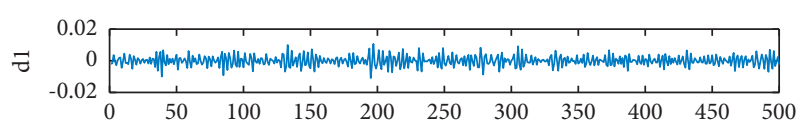

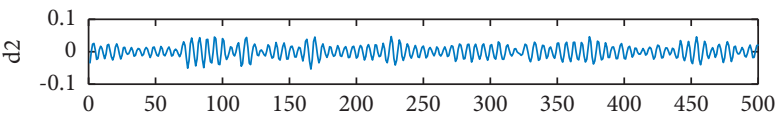

$\Rightarrow \underbrace{0.2}_{0}$

落

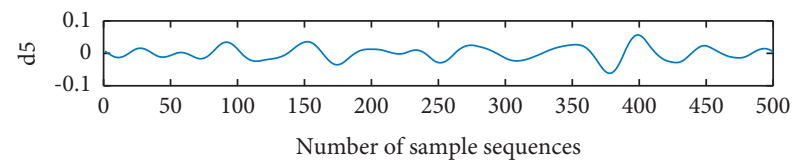

FIGURE 5: 5-layer normal signal wavelet decomposition.
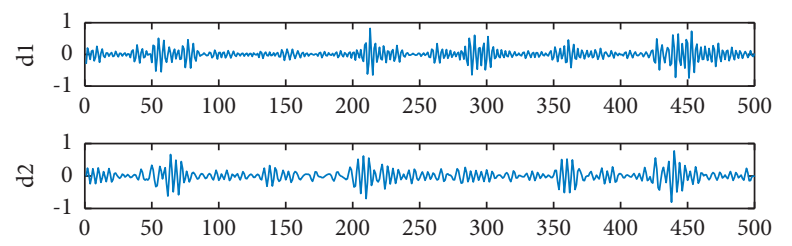

$\Rightarrow \underbrace{0.5}_{-0.5}$

要

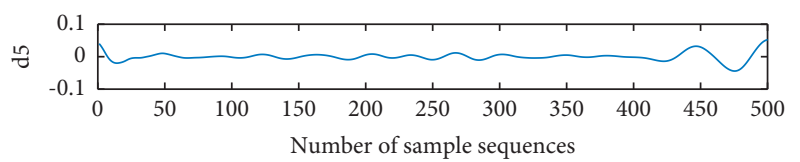

Figure 6: 5-layer fault signal wavelet decomposition.

transformed into stationary signals, signal periodicity was enhanced from the lower to the higher levels, and the effective information weakened and was even lost. When selecting the number of layers of orthogonal wavelet decomposition, it is necessary to consider both stationarity and signal validity to ensure the best classification effect.

5.2. Experiment Steps. First, no OWT was conducted on the data. Feature extraction was not conducted on the original data that was collected when the bearing was normal, and the bearing's inner ring was faulty (0.007 inch), respectively, under 0 HP load, with both cases each having 100 sets. 200 sets of data were used as the input vector of the KNN multiclassifier for testing the fault signal classification, with the parameter $k=3$. The test results are shown in Figure 7. In this figure, the red*represents the normal data under $0 \mathrm{HP}$, and the bluexrepresents the inner ring fault data under $0 \mathrm{HP}$. It can be seen in the figure that the red $*$ and blue $\times$ are crossdistributed along the path, which made it difficult to distinguish fault categories. After that, no feature extraction was performed for the data collected when the bearing was normal, and the bearing's inner ring was faulty (0.007 inch), respectively, under $1 \mathrm{HP}$ load, with both cases each having 10 sets. The 20 sets of data were used as training samples, and the trained KNN multiclassifier mentioned earlier was used to observe the classification results for the fault categories. As can be seen in Figure 7, the red $\bigcirc$ and blue $\square$ represent the normal data and fault data for the $1 \mathrm{HP}$ cases, respectively. It can be seen that the red $\bigcirc$ and blue $\square$ are cross-distributed in the figure, which made it difficult to distinguish between the fault category.

Next, the OWT peak-to-peak feature extraction was conducted on the data.OWT was conducted on 200 sets of data. With 5-layer decomposition and the orthogonal wavelet function being $10 \mathrm{db}$, the peak-to-peak feature vectors of the 5 th and 4th layers were extracted as the input vectors of the OWTKNN multiclassifier to test fault signal classifications. The classification results are shown in Figure 8. In this figure, the red ${ }^{*}$ represents the normal data under $0 \mathrm{HP}$, and the blue $\times$ represents the inner ring fault data under 0 HP. It can be seen that the red* and bluexare well divided into two parts in the figure. The boundaries of these two types of data are clearly defined, the classification effect stands out, and the correct diagnostic rate reaches $100 \%$. After that, another 20 sets of data were collected when the bearing was normal and the bearing's inner ring was faulty ( 0.007 inch), respectively, under 0 HP load, with both cases each having 10 sets. The peak-to-peak feature vectors of the 5 th and 4 th layers were extracted as the sample data to be classified. The trained OWTKNN multiclassifier was used to observe the results of the fault category classification. The red $\bigcirc$ and blue $\square$ in Figure 8 represent the normal data the fault data of the $0 \mathrm{HP}$ case, respectively. As seen in the figure, the two sets of data to be classified and represented by red $\bigcirc$ and blue $\square$, respectively, can be identified completely and correctly in the figure, and the recognition rate is $100 \%$, which means it identifies the fault category very well. Then the 20 sets of data were collected when the bearing was normal and the bearing's inner ring was faulty (0.007 inch), respectively, under $1 \mathrm{HP}$ load, with both cases each having 10 sets. The same method was employed to observe the classification results of the fault category. In Figure 9, the red $\bigcirc$ and blue $\square$ represent the normal data and fault data of the 1HP case, respectively. It can also be seen in the figure that red $\bigcirc$ and blue $\square$ for the two sets of data to be classified can be recognized completely and correctly in the figure with a $100 \%$ recognition rate, which means that unknown fault categories can be well identified.

Again, we changed the orthogonal wavelet feature extraction method. For the 200 sets of data in Figure 7, the peak-to-peak feature vectors of the 4 th and 3 rd layers were extracted as the input vectors of the OWTKNN multiclassifier to test fault signal classification. The classification results are shown in Figure 10. In this figure, the red*represents normal data under $0 \mathrm{HP}$, and the blue$\times$ represents inner ring fault data under $0 \mathrm{HP}$. It can be seen that the red*and bluexare well divided into two parts in the figure, and the two types of data are clearly bounded by farther distances, with a clear classification effect and 100\% correct diagnostic rate. The two sets of data are more 


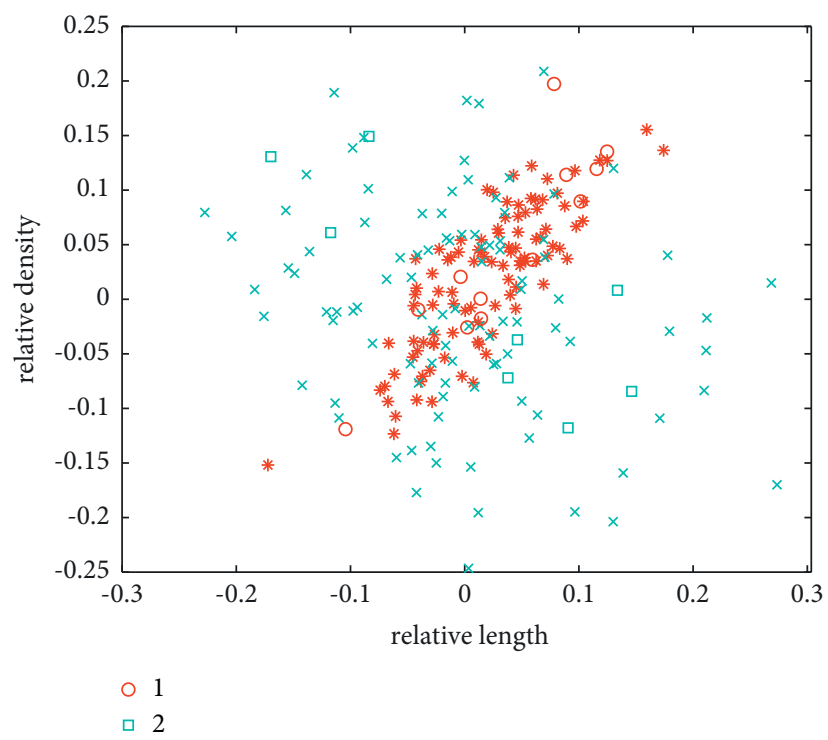

FIGURE 7: KNN when the bearing was normal and subject to 0.007 in. inner ring fault, respectively.

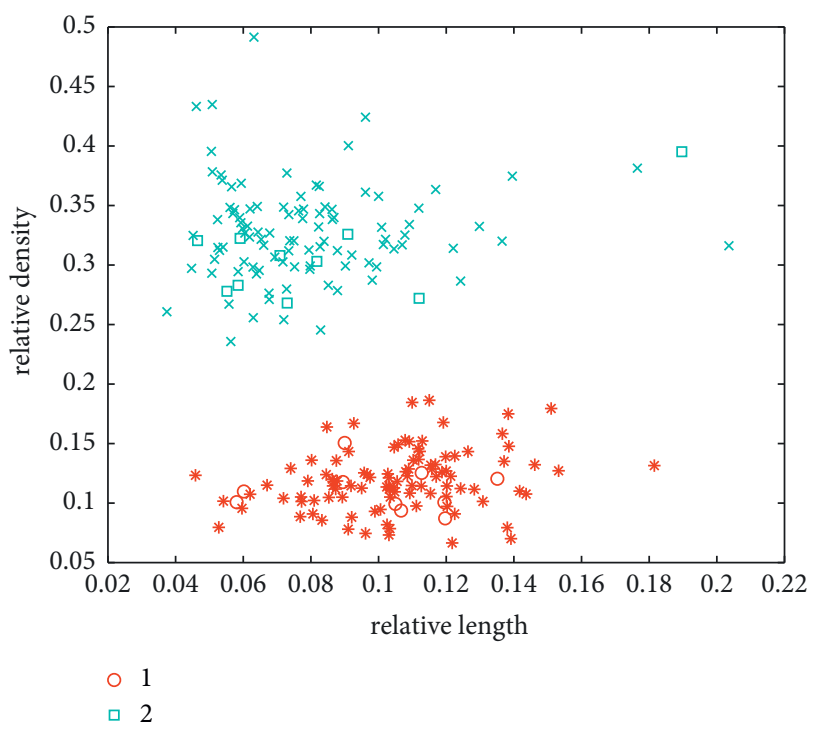

FIGURE 8: OWTKNN of the 4th and 5th layers when the bearing was normal and with an inner ring fault (0.007 in.), respectively.

concentrated. In particular, the normal data samples are concentrated near the vertical coordinate 0.2 unit line, with a better clustering effect. It also shows that after the orthogonal wavelet feature extraction in the 3rd and 4th layers, although regularity was reduced, more information from the original samples was retained, and the classification effect was better. Then the 20 sets of data were collected when the bearing was normal, and the bearing's inner ring was faulty (0.007 inches), respectively, under $1 \mathrm{HP}$ load, with both cases each having 10 sets subject to the same OWT. The peak-topeak feature vectors of the 4 th and 3rd layers were extracted as samples to be classified. The trained OWTKNN multiclassifier was used to observe the results of fault category classification. In Figure 10, the red $\bigcirc$ and blue $\square$ represent the normal data and fault data of the $1 \mathrm{HP}$ case, respectively.

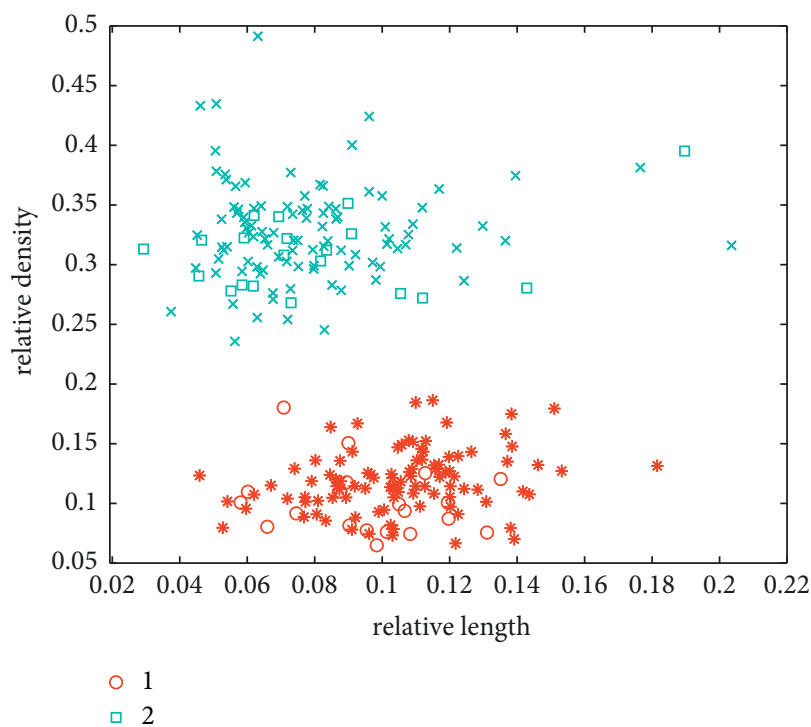

FIgURE 9: OWTKNN of the 4th and 5th layers when the bearing was exhibiting an inner ring fault (0.007 in.) for the $1 \mathrm{HP}$ case.

It can also be seen in the figure that red $\bigcirc$ and blue $\square$ for the two sets of data to be classified can be recognized completely and correctly in the figure with a $100 \%$ recognition rate, which means that the fault category can be recognized very well. The unknown normal samples were more concentrated and clustered more obviously after recognition.

Different feature extraction methods are subsequently compared with Orthogonal Wavelet k-Nearest Neighbors (OWTKNN). Compared with Figure 8, the original $0 \mathrm{HP}$ bearing fault data were collected for every 100 sets of 200 sets of data, which have been used to test different algorithms and feature extraction for fault signal classification, as illustrated in Figure 11. As inferred from the figure (EMD), the red*normal data and the bluexinner circle fault data are divided into two parts in the figure. The boundaries of the two 


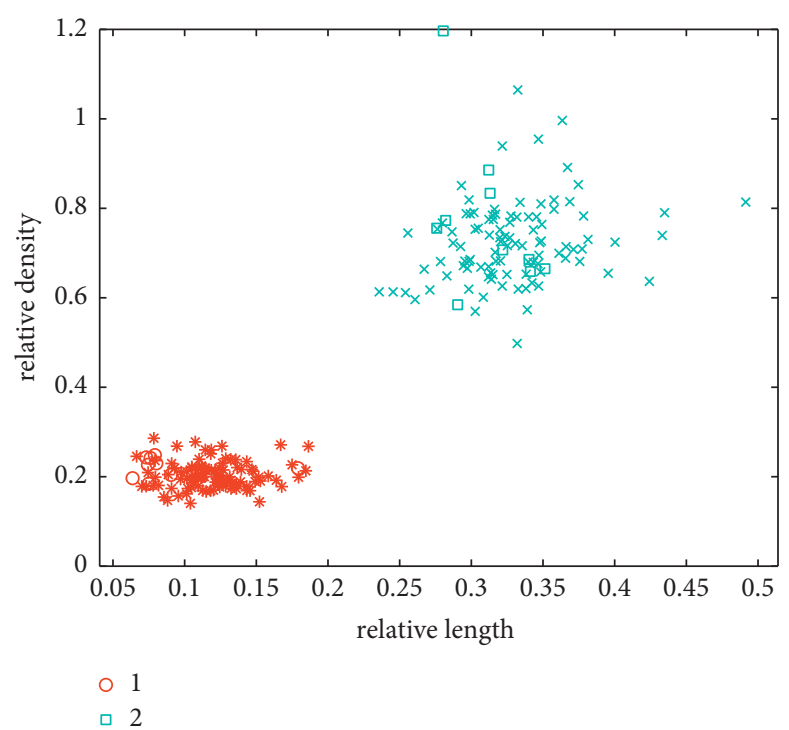

FIgURE 10: OWTKNN of the 3th and 4th layers when the bearing was normal and with an inner ring fault (0.007 in.).
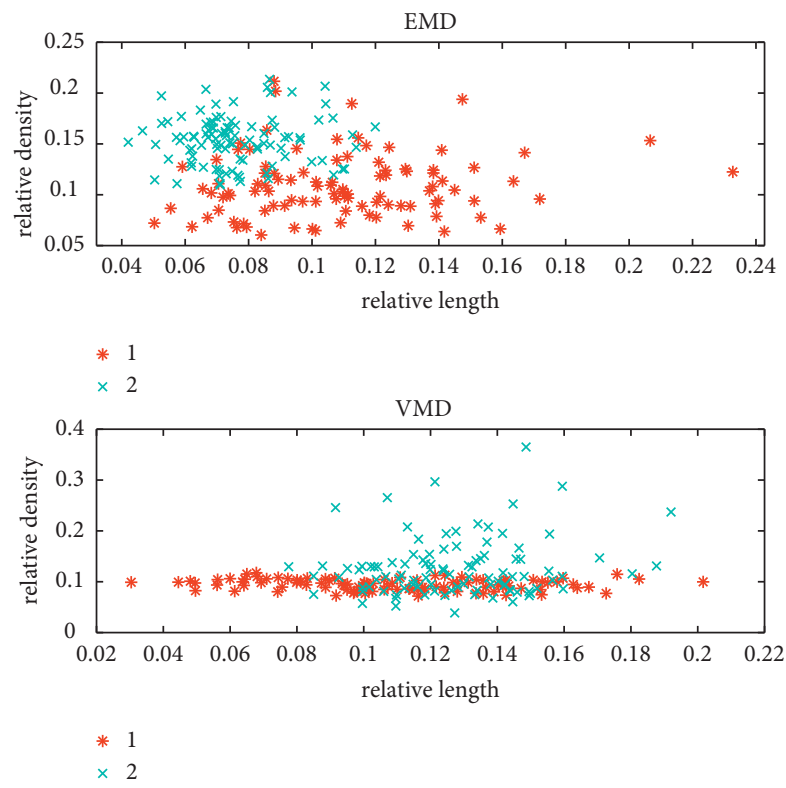

FIGURE 11: EMD and VMD feature extraction were compared with OWTKNN.

types of data are unclear cross-distributed, and the classification effect is not obvious, each of which consists of more than 20 parts. The fault data is misclassified, and the diagnosis accuracy rate is only about $80 \%$. As inferred from the figure (VMD), the boundaries of red $*$ normal data and blue $*$ inner circle fault data are unclear, the two types of data cannot be distinguished, and the diagnostic accuracy rate only reaches $50 \%$. The classification effects of rms, kurtosis, and impulse factor feature extraction are compared in Table 1.

Finally, OWTKNN verification was conducted on the multiple fault signals obtained. Compared to Figure 9, for the 200 sets of data originally collected when the bearing was in faulty under $0 \mathrm{HP}$ load with both cases each having 100 sets, the peak-to-peak feature vectors of the 5 th and 4 th layers were extracted as the input vectors of the OWTKNN multiclassifier to test fault signal classification. 20 sets of data were subsequently collected when the bearing was faulty ( 0.007 inches), under $1 \mathrm{HP}$ load, with both cases each having 10 sets. The peak-to-peak feature vectors of the 5 th and 4 th layers were extracted as samples to be classified. The trained OWTKNN multiclassifier was used to observe the results of the fault category classification, and the fault classification effect is listed in Table 2. 
TABLE 1: Comparison of OWTKNNs for different feature extraction methods.

Algorithm

EMD algorithm feature extraction

VMD algorithm feature extraction

$\mathrm{K}$-centre clustering algorithm (KCA)

Rms extraction

\section{Kurtosis}

Extraction

Impulse factors feature extraction
Every 100 sets of data OWTKNN classification effect 0HP

In Figure 11 (EMD), the red $*$ normal data and the bluexinner circle fault data are divided into two parts in the figure. The boundaries of the two types of data are unclear, cross-distributed, and the classification effect is not obvious. Each consists of over 20 parts, the fault data is misclassified, and the correct rate of diagnosis only reaches $20 \%$.

In Figure $11(\mathrm{VMD})$, the red $*$ normal data and the bluexinner circle fault data are divided into two parts in the figure. The boundary of the two types of data is unclear, cross-distributed, the two types of data cannot be distinguished, and the diagnostic accuracy rate is only about $50 \%$.

In Figure $12(\mathrm{KCA})$, the red $*$ normal data and the blue $\times$ inner circle fault data are divided into two parts in the figure. The boundary of the two types of data is unclear, the cross distribution, and the classification effect is inconspicuous, each with more than 20 parts the fault data is misclassified, and the diagnosis accuracy rate is only about $80 \%$.

In Figure $12(\mathrm{rms})$, the red $*$ normal data and the blue $*$ inner circle fault data are divided into two parts in the figure. The boundary of the two types of data is unclear, and the boundary is cross-distributed, and the classification effect is not obvious, each with 10 multiple fault data are misclassified, and the diagnosis accuracy rate is only about $90 \%$.

In Figure 12 (kurtosis), the red $*$ normal data and the bluexinner circle fault data are divided into two parts in the figure. The boundary of the two types of data is not very clear, cross-distributed. The two types of data cannot be distinguished, and the diagnostic accuracy is only around $30 \%$.

In Figure 12 (impulse factor), the red $*$ normal data and the blue $*$ inner circle fault data are divided into two parts in the figure. The boundary of the two types of data is unclear, cross-distributed, the two types of data cannot be distinguished, and the diagnostic accuracy rate only reaches about $30 \%$.
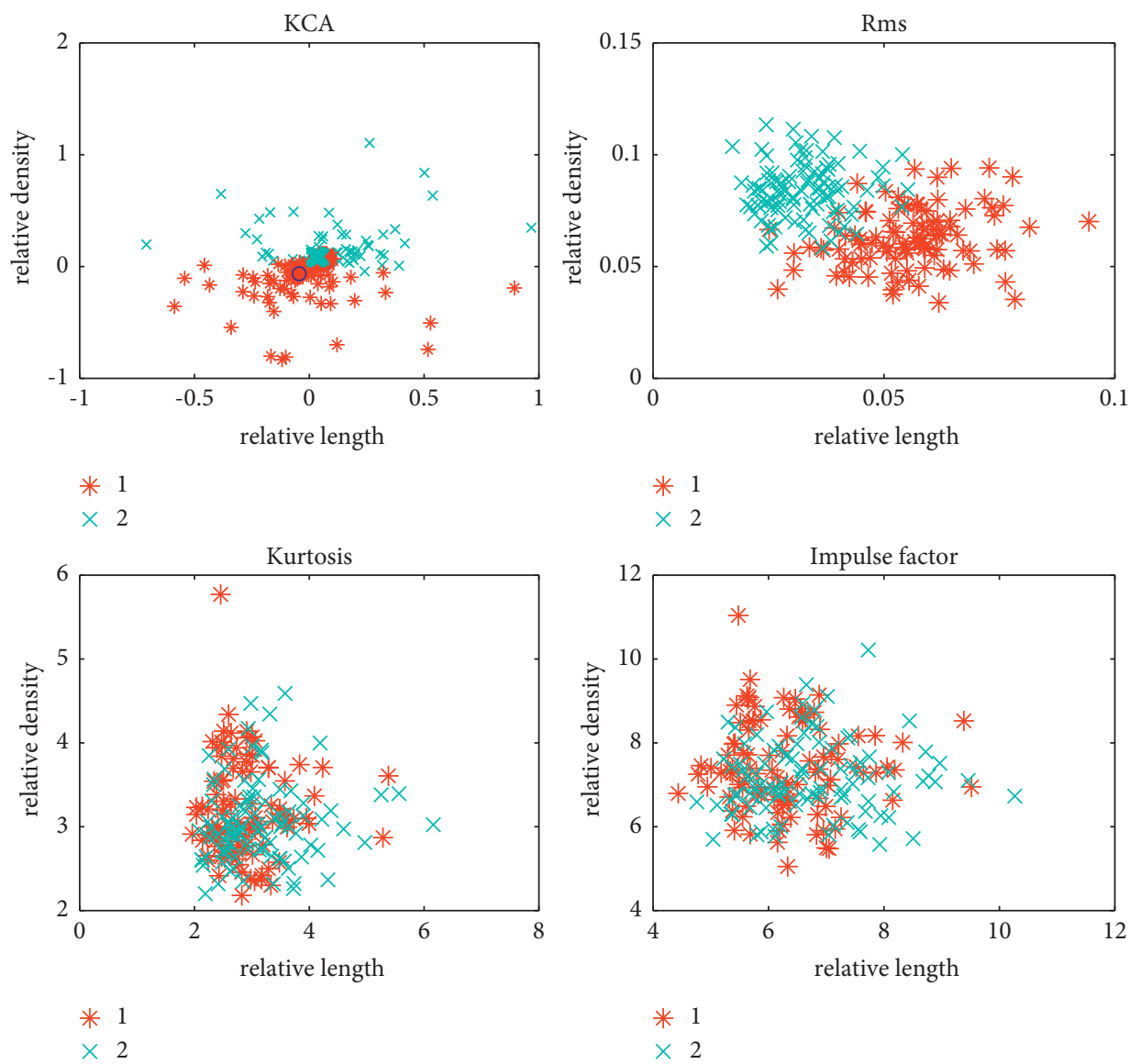

FIgURE 12: KCA, Rms, Kurtosis, Impulse factor feature extraction, and comparison of OWTKNN. 
TABLE 2: Comparison of OWTKNNs for multiple fault signals.

\begin{tabular}{|c|c|c|c|}
\hline Fault 1 & Fault 2 & $\begin{array}{l}\text { OWTKNN classification effect of every } 100 \text { sets of } \\
\text { data for the } 0 \mathrm{HP} \text { case }\end{array}$ & $\begin{array}{l}\text { OWTKNN multiclassification effect for every } 10 \\
\text { datasets for the } 1 \mathrm{HP} \text { case }\end{array}$ \\
\hline $\begin{array}{l}\text { The bearing } \\
\text { was normal }\end{array}$ & $\begin{array}{l}\text { Inner ring } \\
0.007 \text { in. }\end{array}$ & $\begin{array}{l}\text { In Figure } 9 \text {, the red } * \text { and blue } \times \text { for the normal data } \\
\text { from the bearing and fault data of the inner ring are } \\
\text { smoothly divided into two parts. The boundary of } \\
\text { the two types of data is clear, with obvious } \\
\text { classification effects and a correct diagnosis rate of } \\
100 \% \text {. }\end{array}$ & $\begin{array}{l}\text { In Figure 9, for the two sets of classified data, the normal } \\
\text { bearing and inner ring fault represented by red } \bigcirc \text { and } \\
\text { blue } \square \text {, respectively, can be correctly identified with a } \\
\text { recognition rate of } 100 \% \text {. This means that the fault } \\
\text { category can be effectively recognized. }\end{array}$ \\
\hline $\begin{array}{l}\text { Inner ring } \\
0.007 \mathrm{in.}\end{array}$ & $\begin{array}{l}\text { Ball } 0.007 \\
\quad \text { in. }\end{array}$ & $\begin{array}{l}\text { In Figure } 13 \text {, the red } * \text { and blue } \times \text { for the fault data of } \\
\text { the inner ring and ball are well divided into two } \\
\text { parts. The boundary of the two types of fault data is } \\
\text { clear except for } 2 \text { data points in close proximity, with } \\
\text { obvious classification effects and a correct diagnosis } \\
\text { rate exceeding } 99 \% \text {. }\end{array}$ & $\begin{array}{l}\text { In Figure } 13 \text {, for the two sets of data to be classified, the } \\
\text { faults of the inner ring and the ball represented by red } \bigcirc \\
\text { and blue } \square \text {, respectively, can be completely correctly } \\
\text { identified with a recognition rate of } 100 \% \text {. This means } \\
\text { that the fault category can be well recognized. }\end{array}$ \\
\hline $\begin{array}{l}\text { Inner ring } \\
0.007 \text { in. }\end{array}$ & $\begin{array}{l}\text { Ball } 0.014 \\
\quad \text { in. }\end{array}$ & $\begin{array}{l}\text { In Figure } 14 \text {, the red } * \text { and blue } \times \text { for the fault data of } \\
\text { the inner ring and ball are well divided into two } \\
\text { parts. The boundary of the two types of fault data is } \\
\text { clear except that } 2 \text { blue } \times \text { ball fault data points are } \\
\text { improperly classified. The classification effect is } \\
\text { obvious, and the correct diagnosis rate exceeds } 98 \% \text {. }\end{array}$ & $\begin{array}{l}\text { In Figure } 14 \text {, to classify the two sets of data to, the faults } \\
\text { of the inner ring and the ball represented by red } \bigcirc \text { and } \\
\text { blue } \square \text {, respectively, can be correctly identified with a } \\
\text { recognition rate of } 100 \% \text {. This means that the fault } \\
\text { category can be recognized quite well. }\end{array}$ \\
\hline $\begin{array}{l}\text { Inner ring } \\
0.007 \text { in. }\end{array}$ & $\begin{array}{l}\text { Inner ring } \\
0.014 \text { in }\end{array}$ & $\begin{array}{l}\text { In Figure } 15 \text {, the red } * \text { and blue } \times \text { for the fault data of } \\
\text { the inner ring and ball are divided into two parts } \\
\text { quite well. For the data points, except for } 2 \text { red } * \\
\text { inner circle faults ( } 0.007 \text { in.) and } 5 \text { blue } \times \text { inner circle } \\
\text { faults ( } 0.014 \text { in.) which are improperly classified, the } \\
\text { other data boundaries are clearly defined, the } \\
\text { classification effect is obvious, and the correct } \\
\text { diagnosis rate over } 95 \% \text {. }\end{array}$ & $\begin{array}{l}\text { In Figure } 15 \text {, to classify the two sets of data to, the fault } \\
(0.007 \text { in.) and the fault }(0.014 \text { in.) of the inner ring } \\
\text { represented by red } \bigcirc \text { and blue } \square \text {, respectively, can be } \\
\text { correctly identified with a recognition rate of } 100 \% \text {. This } \\
\text { means that the fault category can be effectively } \\
\text { recognized. }\end{array}$ \\
\hline
\end{tabular}

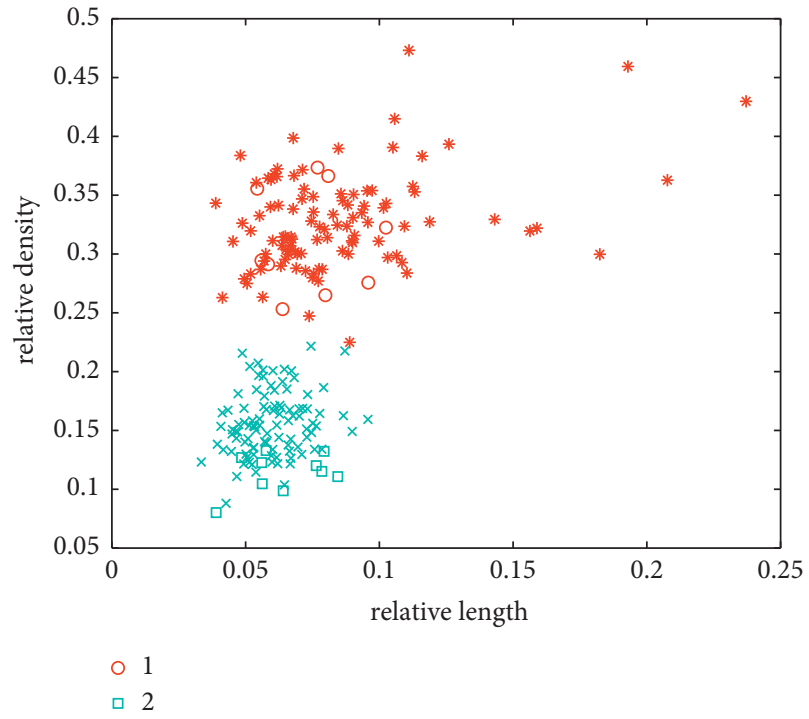

FIGURE 13: OWTKNN of 0.007 in. inner ring fault and 0.007 in. ball fault.

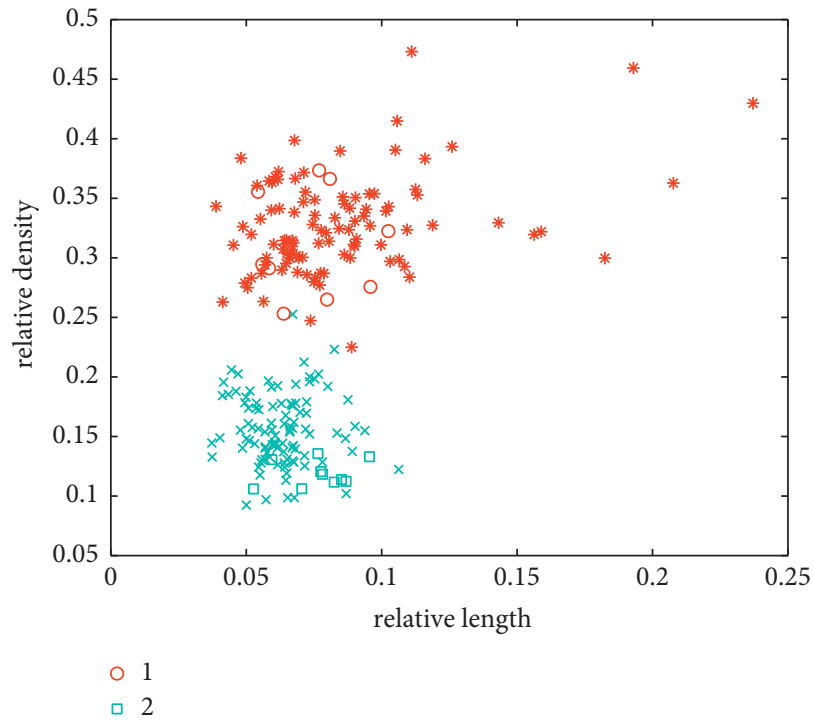

FIGURE 14: OWTKNN of 0.007 in. inner ring fault and 0.014 in. ball fault. 


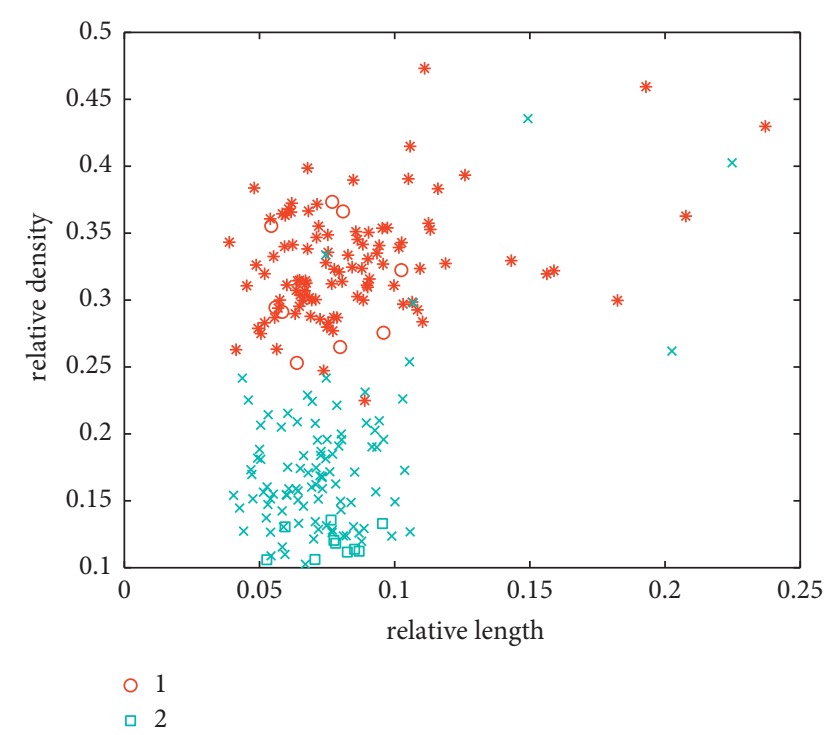

FIGURE 15: OWTKNN of 0.007 in. and 0.014 in. inner ring faults.

\section{Conclusion}

A method (OWTKNN) for peak-to-peak feature extraction by OWT and fault diagnosis and identification by the KNN multiclassifier was proposed. The method fully utilizes the wavelet decomposition to handle rough signals and the KNN algorithm to classify mechanical fault data signals efficiently with limited surrounding nearest neighbors and extracts peak-to-peak feature information through wavelet decomposition. It can be verified that the proposed method is effective for diagnosing bearing faults by classifying the data for normal bearings and inner ring faults, inner ring faults, and ball faults, and by comparing them with the KCA method. The conclusions drawn are as follows:

(1) This method extracts peak-to-peak feature information by wavelet decomposition. Compared with the KNN methods lacking feature extraction, OWTKNN is more efficient for classifying fault data, with a recognition rate of $100 \%$.

(2) This method can correctly identify and classify the inner ring and ball, inner ring, and inner ring fault signals of various bearing faults, with a total recognition rate exceeding 95\%.

(3) By changing the features in different orthogonal wavelet layers, the classification effect witnessed improvements, the classification time was shortened, and the efficiency and accuracy of intelligent diagnosis were significantly improved.

(4) A comparison with the KCA method also proved the effectiveness of the proposed method, and the recognition rate for the KCA method only exceeded $80 \%$.

(5) This method fully utilizes the features that allow wavelet decomposition to properly handle nonstationary signals, and the KNN method can classify mechanical fault data signals with limited neighbors, thereby improving the classification effect by changing the features in different orthogonal wavelet layers

(6) Only the fault data can be clustered properly. If the input fault samples have a large capacity while other capacities are small, a possibility exists that largecapacity samples will account for the majority, leading to misclassification.

(7) The proposed algorithm is more suitable for automatically classifying class domains with large sample sizes, while those with small sample sizes are prone to misclassification. If this fault misclassification were to be overcome, the sample feature extraction method would then be continually improved to allow fault samples to be more representative.

OWTKNN is widely used in online fault diagnosis, artificial intelligence, data processing, and other fields, which can substantially improve fault diagnosis and data classification efficiencies. Future research will focus on improving fault diagnosis techniques based on signal analysis of vibrations, acoustic signals, thermal signals, etc. Various types of faults and machines will be analyzed to develop improved mechanical fault diagnosis methods.

\section{Data Availability}

The data used to support this study are included within the article.

\section{Conflicts of Interest}

The authors declare that they have no conflicts of interest.

\section{Authors' Contributions}

Li Weipeng performed the numerical simulation. Li Weipeng and Cao Yan analyzed the theoretical study, analyzed the results of numerical simulation, and prepared the manuscript. Li Lijuan and Hou Siyu reviewed the manuscript.

\section{Acknowledgments}

This work was supported by a grant from Shaanxi Province Innovation Capacity Support Program 2018 under TD-036 and Shaanxi Province Key R\&D Program 2019 under GY125.

\section{References}

[1] S. W. Fei, "The hybrid method of VMD-PSR-SVD and improved binary PSO-KNN for fault diagnosis of bearing," Shock and Vibration, vol. 2019, Article ID 4954920, 7 pages, 2019.

[2] A. Moosavian, H. Ahmadi, A. Tabatabaeefar, and M. Khazaee, "Comparison of two classifiers; K-nearest neighbor and artificial neural network, for fault diagnosis on a main engine journal-bearing," Shock and Vibration, vol. 20, p. 10, Article ID 360236, 2013.

[3] X. Yu, W. Chen, C. L. Wu et al., "Rolling bearing fault diagnosis based on domain adaptation and preferred feature 
selection under variable working conditions," Shock and Vibration, vol. 2021, Article ID 8843124, 27 pages, 2021.

[4] P. B. Zheng and J. W. Zhang, "Application of variational mode decomposition and k-nearest neighbor algorithm in the quantitative nondestructive testing of wire ropes," Shock and Vibration, vol. 2019, Article ID 9828536, 14 pages, 2019.

[5] K. Sangsik, L. M. Hee, W. Theanchai et al., "Human sensorinspired supervised machine learning of smartphone-based paper microfluidic analysis for bacterial species classification," Biosensors and Bioelectronics, vol. 188, 2021.

[6] C. G. Kibbey Tohren, J. Rafal, and M. Oarroll Deni, "Source allocation of polyfluoroalkyl substances (PFAS) with supervised machine learning:Classification performance and the role of feature selection in an expanded dataset," Chemosphere, vol. 275, Article ID 130124, 2021.

[7] J. T. Lu, W. W. Qian, S. M. Li, and S. Cui, "Enhanced $\mathrm{K}$-nearest neighbor for intelligent fault diagnosis of rotating machinery," Applied Sciences, vol. 11, no. 3, p. 919, 2021.

[8] W. Qian, S. Li, and J. Wang, "An intelligent fault diagnosis framework for raw vibration signals: adaptiveoverlapping convolutional neural network," Meas. Sci. Technol. Measurement Science and Technology, vol. 29, no. 9, 2018.

[9] F. Gorunescu and S. Belciug, "Intelligent decision support systems in automated medical diagnosis," Advances in Biomedical Informatic, vol. 137, pp. 161-186, 2018.

[10] D. T. Hoang and H. J. Kang, "A survey on deep learning based bearing fault diagnosis," Neurocomputing, vol. 335, pp. 327-335, 2019.

[11] A. Glowacz, "Ventilation diagnosis of angle grinder using thermal imaging," Sensors, vol. 212853 pages, 2021.

[12] O. AlShorman, M. Irfan, N. Saad, D. Zhen, N. Haider, and A. Glowacz, "A review of artificial intelligence methods for condition monitoring and fault diagnosis of rolling element bearings for induction motor," Shock and Vibration, vol. 202020 pages, 2020.

[13] A. Glowacz, R. Tadeusiewicz, and S. Legutko, "Fault diagnosis of angle grinders and electric impact drills using acoustic signals," Applied Acoustics, vol. 179, 2021.

[14] J. W. Jiang and Y. H. Hu, "Fault diagnosis of diesel engines based on wavelet packet energy spectrum feature extraction and fuzzy entropy feature selection," Journal of Vibration and Shock, vol. 39, pp. 273-277+298, 2020.

[15] X. B. Liu, M. M. Zhang, and J. C. Tu, "A wavelet cluster algorithm based on breadth-first-search(BFS)," Journal of Vibration and Shock, vol. 35, no. 15, pp. 178-183, 2016.

[16] H. Zhao, H. Liu, Y. Jin, X. Ding, and W. Deng, "Feature extraction for data-driven remaining useful life prediction of rolling bearings," IEEE Transactions on Instrumentation and Measurement, vol. 70, no. 3511910, pp. 1-10, 2021.

[17] X. Cai, H. M. Zhao, and S. F. Shang, "An improved quantuminspired cooperative co-evolution algorithm with mulistrategy and its application," Expert Systems with Applications, vol. 171, no. 114629 .

[18] P. Chen, X. Q. Zhao, and H. M. Jiang, "A new method of fault feature extraction based on hierarchical dispersion entropy," Shock and Vibration, vol. 2021, Article ID 8824901, 11 pages, 2021.

[19] C. W. Fei and G. C. Bai, "Wavelet correlation feature scale entropy and fuzzy support vector machine approach for aeroengine whole-body vibration fault diagnosis," Shock and Vibration, vol. 20, pp. 341-349, 2013.

[20] K. S. Lu, C. B. Guang, and W. F. Cheng, "Probabilistic LCF life assessment for turbine discs with DC strategy-based wavelet neural network regression," International Journal of Fatigue, vol. 119, pp. 204-219, 2019.
[21] Y. N. Pan and J. Chen, "Wavelet package-support vector data description applied in bearing performance degradation assessment," Journal of Vibration and Shock, vol. 28, no. 14, pp. 164-167, 2009.

[22] X. B. Liu, Z. D. Han, W. Q. Shao, and H. Y. Zuo, "A doublegrid correction wavelet clustering algorithm based on hash function," Journal of Vibration and Shock, vol. 37, no. 21, pp. 267-272+280, 2018.

[23] P. Chayashree and G. Amrita, "Adaptive thresholding technique based classification of red blood cell and sickle cell using Naïve Bayes Classifier and K-nearest neighbor classifier," Biomedical Signal Processing and Control, vol. 68, 2021.

[24] W. J. Huang and K. W. Wen, "Fast KNN classification algorithm based an partial distance search," Electronics Letters, vol. 34, no. 21, pp. 2062-2063, 1998.

[25] Y. Gao, X. Liu, H. Huang, and J. Xiang, "A hybrid of FEM simulations and generative adversarial networks to classify faults in rotor-bearing systems," ISA Transactions, vol. 108, 2020.

[26] X. Ran, X. Zhou, M. Lei, W. Tepsan, and W. Deng, "A novel $\mathrm{K}$-means clustering algorithm with a noise algorithm for capturing urban hotspots," Applied Sciences, vol. 11, Article ID 11202, 2021.

[27] Q. Wu and Z. MengChu, "Self-paced dynamic infinite mixture modelfor fatigue evaluation of pilots' brains," IEEE Transactions on Cybernetics, pp. 1-16, 2020.

[28] H. J. Cui, Y. Guan, and H. Y. Chen, “A novel advancing signal processing method based on coupled multi-stable stochastic resonance for fault detection," Applied Sciences, vol. 11, p. $5385,2021$.

[29] Z. H. Zhang, F. Min, and G. S. Chen, "Tri-partition state alphabet-based sequential pattern for multivariate time series," Cognitive Computation, pp. 1-19, 2021.

[30] H. J. Cui, Y. Guan, and H. Y. Chen, "Rolling element fault diagnosis based on VMD and sensitivity MCKD," Digital Object Identifier, p. 1, 2021.

[31] X. J. Li, "Research on K-nearest neighbor algorihtm in classification," SCIENCE \& TECHNOLOGY INFORMATION, vol. 383, no. 31, p. 81, 2009.

[32] Z. Y. Zhang, Y. H. Huang, and H. H. Wang, "A new KNN classification approach," Computer Science, vol. 35, no. 3, pp. 170-172, 2008.

[33] G. H. Huang, F. Yin, and J. L. Feng, "An improved KNN algorithm based on cross validation and distance weighting," Journal of Southwest University for Nationalities (Natrual Science Edition), vol. 46, no. 2, pp. 172-177, 2020.

[34] W. P. Li, Y. Cao, L. J. Li, and S. Y. Hou, "Orthogonal wavelet transform KCA in fault diagnosis," Journal of Vibration and Shock, vol. 40, no. 7, pp. 291-296, 2021.

[35] H. D. Shao, H. K. Jiang, K. Zhao, D. Wen, and X. Li, "A novel tracking deep wavelet auto-encoder method for intelligent fault diagnosis of electric locomotive bearings," Mechanical Systems and Signal Processing, vol. 110, pp. 193-209, 2018.

[36] F. Kong and J. Li, "Periodic generalized harmonic wavelet transformation and reconstruction," Journal of Vibration and Shock, vol. 32, no. 7, pp. 24-29, 2013.

[37] S. W. Li, D. L. Zhang, X. Y. Huang, and P. Chen, "Application of data feature selection and classification in mechanical fault diagnosis," Journal of Vibration and Shock, vol. 39, no. 2, pp. 218-222, 2020.

[38] L. J. Li, J. Han, W. P. Li, and W. W. F. Kong, "Applying orthogonal wavelet transform-SVDD to evaluating performance of bearing," Mechanical Science and Technology, vol. 31, no. 7, pp. 1201-1204, 2012. 
[39] L. J. Wan, K. Gong, and G. Zhang, "An efficient rolling bearing fault diagnosis method based on spark and improved," Random Forest Algorithm, no. 4, pp. 1-18, Article ID 3063929, 2016.

[40] S. S. Wu, X. Y. Jing, and Q. H. Zhang, "Prediction consistency guided convolutional neural networks for cross-domain bearing fault diagnosis," IEEE Access, vol. 8, Article ID 3005422, 2020. 